\title{
Identifying the key genes and microRNAs in colorectal cancer liver metastasis by bioinformatics analysis and in vitro experiments
}

\author{
TAO ZHANG $^{1 *}$, JIANRONG GUO $^{1 *}$, JIAN GU $^{1}$, ZHENG WANG $^{1,2}$, GUOBIN WANG $^{1}$, \\ HUILI LI ${ }^{1}$ and JILIANG WANG ${ }^{1}$ \\ ${ }^{1}$ Department of Gastrointestinal Surgery; ${ }^{2}$ Research Center for Tissue Engineering and Regenerative Medicine, Union Hospital, \\ Tongji Medical College, Huazhong University of Science and Technology, Wuhan 430022, China
}

Received May 22, 2018; Accepted October 16, 2018

DOI: $10.3892 /$ or.2018.6840

\begin{abstract}
Colorectal cancer (CRC) is one of the principal causes of cancer-associated mortality worldwide. The high incidence of liver metastasis is the leading risk factor of mortality in patients with $\mathrm{CRC}$, and the mechanisms of CRC liver metastasis are poorly understood. In the present study, 7 datasets, including 3 gene expression profile datasets and 4 microRNA (miRNA) expression profile datasets were downloaded from the NCBI Gene Expression Omnibus (GEO) database to identify potential key genes and miRNAs, which may be candidate biomarkers for CRC liver metastasis. Differentially expressed (DE) genes (DEGs) and DE miRNAs of primary CRC tumor tissues and liver metastatic CRC tumor tissues were selected using the GEO2R tool. Gene Ontology and Kyoto Encyclopedia of Gene and Genome pathway enrichment analyses were conducted using the Database for Annotation, Visualization and Integrated Discovery online database. Furthermore, Cytoscape with cytoHubba and the Molecular Complex Detection (MCODE) plug-in were used to visualize a protein-protein interaction (PPI) network for these DEGs, and to screen hub genes and gene modules in the PPI network. In addition, the online databases, TargetScan, miRanda, PITA, miRWalk and miRDB, were used to identify the target genes of the DE miRNAs. In the present study, 141 DEGs (97 upregulated and 44 downregulated) and 3 DE miRNAs ( 2 upregulated and 1 downregulated) were screened from the 3 gene expression microarray datasets and 4 miRNA expression microarray datasets, respectively. In total, 10 hub genes with a high degree
\end{abstract}

Correspondence to: Dr Huili Li or Professor Jiliang Wang, Department of Gastrointestinal Surgery, Union Hospital, Tongji Medical College, Huazhong University of Science and Technology, Wuhan 430022, China

E-mail: huili_li@hust.edu.cn

E-mail: jiliang_wang@hust.edu.cn

${ }^{*}$ Contributed equally

Key words: colorectal cancer, liver metastasis, bioinformatics analysis, differentially expressed genes, differentially expressed microRNAs, microRNA-885 of connectivity were selected from the PPI network, including albumin (ALB), coagulation factor II (F2), thrombin, apolipoprotein $\mathrm{H}(\mathrm{APOH})$, serpin family $\mathrm{C}$ member 1 (SERPINC1), apolipoprotein A1 (APOA1), $\alpha$-1-microglobulin/bikunin precursor (AMBP), apolipoprotein C3 (APOC3), plasminogen (PLG), $\alpha-2$ HS glycoprotein (AHSG) and apolipoprotein B (APOB). The most important module was detected in the PPI network using the MCODE plug-in. A total of 20 DEGs were identified to be potential target genes of these DE miRNAs, and novel miRNA-DEGs regulatory axes were constructed. In vitro experiments were performed to demonstrate that miR-885 promoted CRC cell migration by, at least partially, decreasing the expression of von Willebrand factor (vWF) and insulin-like growth factor binding protein 5 (IGFBP5). In conclusion, by using integrated bioinformatics analysis and in vitro experiments, key candidate genes were identified and novel miRNA-mRNA regulatory axes in CRC liver metastasis were constructed, which may improve understanding of the molecular mechanisms underlying CRC liver metastasis.

\section{Introduction}

Colorectal cancer (CRC) is one of the most common malignancies associated with high recurrence incidence and poor prognosis (1). Although diagnostic methods and comprehensive treatment have been developed over the past decades, the mortality rate of CRC remains as the second and third highest-ranked cancer in male and female patients, respectively, in the USA (2) and is ranked fifth in China (3). The liver is the most common metastatic site of CRC. It is estimated that $15-25 \%$ of patients with CRC have synchronous liver metastasis, which is detected at the time of the initial diagnosis. Another $20 \%$ of patients with CRC have heterochronous liver metastases, which typically develops following resection of the primary tumor (4). Liver metastasis is one of the leading causes of cancer-associated mortalities in patients with CRC. The 5-year overall survival rate of patients with liver metastatic CRC is only $25-40 \%$, which is markedly lower compared with patients without liver metastasis (69.5-95.7\%) (5). Since the morbidity and mortality of liver metastatic CRC remains high, identifying the causes, molecular mechanisms and molecular biomarkers for early prediction and personalized therapy is important and urgently required. 
MicroRNAs (miRNAs) are short (21-25 nucleotide) non-coding RNAs involved in the translational and post-transcriptional regulation of gene expression. miRNAs promote the translational repression or degradation of their targets by binding to the complementary sequences in the 3'-untranslated region of target mRNAs, thus affecting multiple signaling pathways (6). Recent studies have suggested the critical role of miRNAs (miRs) in various physiological and pathological processes in CRC, including metastasis (7). For example, miR-21, miR-31, miR-103, miR-107 and miR-122 have been implicated in tumor local invasion; and the miR-17-91 cluster (miR-17, miR-18a, miR-19a/b, miR-20a and miR-92a), miR-21, miR-126 and miR-155 may be involved in tumor intravasation, circulation and extravasation (7). Therefore, identifying diagnostic and therapeutic miRNAs could additionally be of great clinical significance.

The microarray technique is increasingly being used for life science research purposes. Bioinformatics data-mining of gene and non-coding RNA expression microarray data is a useful tool for identifying novel significant genes and non-coding RNAs involved in the pathogenesis of diseases, providing valuable insights and a basis for further novel research (8). In the present study, 3 gene expression profile datasets, GSE41258 (9), GSE68468 (10) and GSE81582-GPL15207 (11), and 4miRNA expression profile datasets, GSE35834 (12), GSE44121, GSE72199 (13) and GSE81582-GPL16384 (11) were downloaded from the Gene Expression Omnibus (GEO). The GEO2R online tool was used to identify the differentially expressed (DE) genes (DEGs) and DE miRNAs of primary CRC tumor tissues and liver metastatic CRC tumor tissues. Gene Ontology (GO) function and Kyoto Encyclopedia of Genes and Genomes (KEGG) pathways enrichment analyses were conducted for the DEGs. A protein-protein interaction (PPI) network of the DEGs was constructed and the hub genes with a higher degree of connectivity were selected. Target genes of these DE miRNAs were predicted using online datasets, and novel miRNA-mRNA regulatory axes were constructed, which were expected to serve vital roles in the liver metastasis of CRC, and to thus serve as novel biomarkers for diagnosis and therapy of CRC.

In the present study, $3 \mathrm{DE}$ miRNAs were identified, namely miR-10b, miR-122 and miR-885. Among them, the function and mechanism of miR-885 in tumorigenesis and progression remain unclear. Previous studies revealed that miR- 885 served as a tumor suppressor by reducing matrix metalloproteinase- 9 (MMP-9) expression (14), repressing cyclin-dependent kinase 2 and DNA replication licensing factor MCM5 expression (15) and inhibiting Wnt/ $\beta$-catenin signaling (16). A recent study observed that miR-885 was overexpressed in CRC liver metastasis and induced CRC metastasis by targeting cytoplasmic polyadenylation element-binding protein 2 (CPEB2) (17). In the present study, the results demonstrated that miR-885 promoted metastasis of CRC in vitro, and that von Willebrand factor (vWF) and insulin-like growth factor binding protein 5 (IGFBP5) are potential novel target genes of miR-885, indicating that the miR-885/vWF and miR-885/IGFBP5 axes may serve roles in the metastasis of CRC.

\section{Materials and methods}

Microarray data. The bioinformatics analysis was conducted following the procedure presented in Fig. 1. The primary
CRC tumor tissues and CRC liver metastatic tumor tissue gene expression profiles of GSE41258, GSE68468 and GSE81582-GPL15207, and the miRNA expression profiles of GSE35834, GSE44121, GSE72199, and GSE81582-GPL16384, were obtained from NCBI-GEO (http://www.ncbi.nlm. nih.gov/geo), a free database of microarray profiles and next-generation sequencing. The microarray data of GSE41258 and GSE68468 were based on the GPL96 platform ([HG-U133A] Affymetrix Human Genome U133A Array), including 186 primary CRC tumor tissues and 47 CRC liver metastatic tumor tissues. GSE81582-GPL15207 was based on the GPL15207 platform ([PrimeView] Affymetrix Human Gene Expression Array), including 23 primary CRC tumor tissues and 19 CRC liver metastatic tumor tissues. GSE35834 was based on the GPL8786 platform [(miRNA-1) Affymetrix Multispecies miRNA-1 Array], including 31 primary CRC tumor tissues and 24 CRC liver metastatic tumor tissues. GSE44121 was based on the GPL16231 platform (Nanostring nCounter Human microRNA Expression Platform), including 9 primary CRC tumor tissues and 9 CRC liver metastatic tumor tissues. GSE72199 was based on the GPL15018 platform [Agilent-031181 Unrestricted_Human_miRNA_ V16.0_Microarray 030840 (Feature Number version)], including 28 primary CRC tumor tissues and 8 CRC liver metastatic tumor tissues. GSE81582-GPL16384 was based on GPL16384 [(miRNA-3) Affymetrix Multispecies miRNA-3 Array], including 23 primary CRC tumor tissues and 19 CRC liver metastatic tumor tissues.

Identification of DEGs and DE miRNAs. The GEO2R online tool (https://www.ncbi.nlm.nih.gov/geo/geo2r/), which allows users to compare two or more groups of samples in a GEO series, was applied to detect DEGs and DE miRNAs of primary CRC tumors and CRC liver metastatic tumors. $\mathrm{P}<0.05$ and llog fold change (FC) $\mid \geq 1$ were set as the cut-off criteria.

GO functional analysis and KEGG pathway enrichment analysis of DEGs. GO functional analysis is a useful method for annotating genes and identifying characteristic biological attributes for high-throughput genome or transcriptome data (18). KEGG incorporates a wide range of databases, including those on genomes, biological pathways, diseases, drugs and chemical substances (19). The GO functional analysis and KEGG pathway enrichment analysis of DEGs were conducted using Database for Annotation, Visualization and Integrated Discovery (DAVID; https://david.ncifcrf.gov/), an online bioinformatics database that aims to provide tools for the functional interpretation of genes or proteins (20). $\mathrm{P}<0.05$ was set as the cut-off criterion.

PPI network and module analysis. The online database STRING (http://string-db.org) (21) was used to develop a PPI network. Cytoscape software (22) was used to construct a PPI network and analyze the interactions of the DEGs. The cytoHubba plug-in was used to identify hub genes. The Molecular Complex Detection (MCODE) plug-in was used to screen modules of the PPI network in Cytoscape with a degree cut-off $=2$, node score cut-off $=0.2$, $\mathrm{k}$-core $=2$ and $\max$ depth $=100$. The KEGG pathway enrichment analysis of genes in the top module was performed using DAVID. 


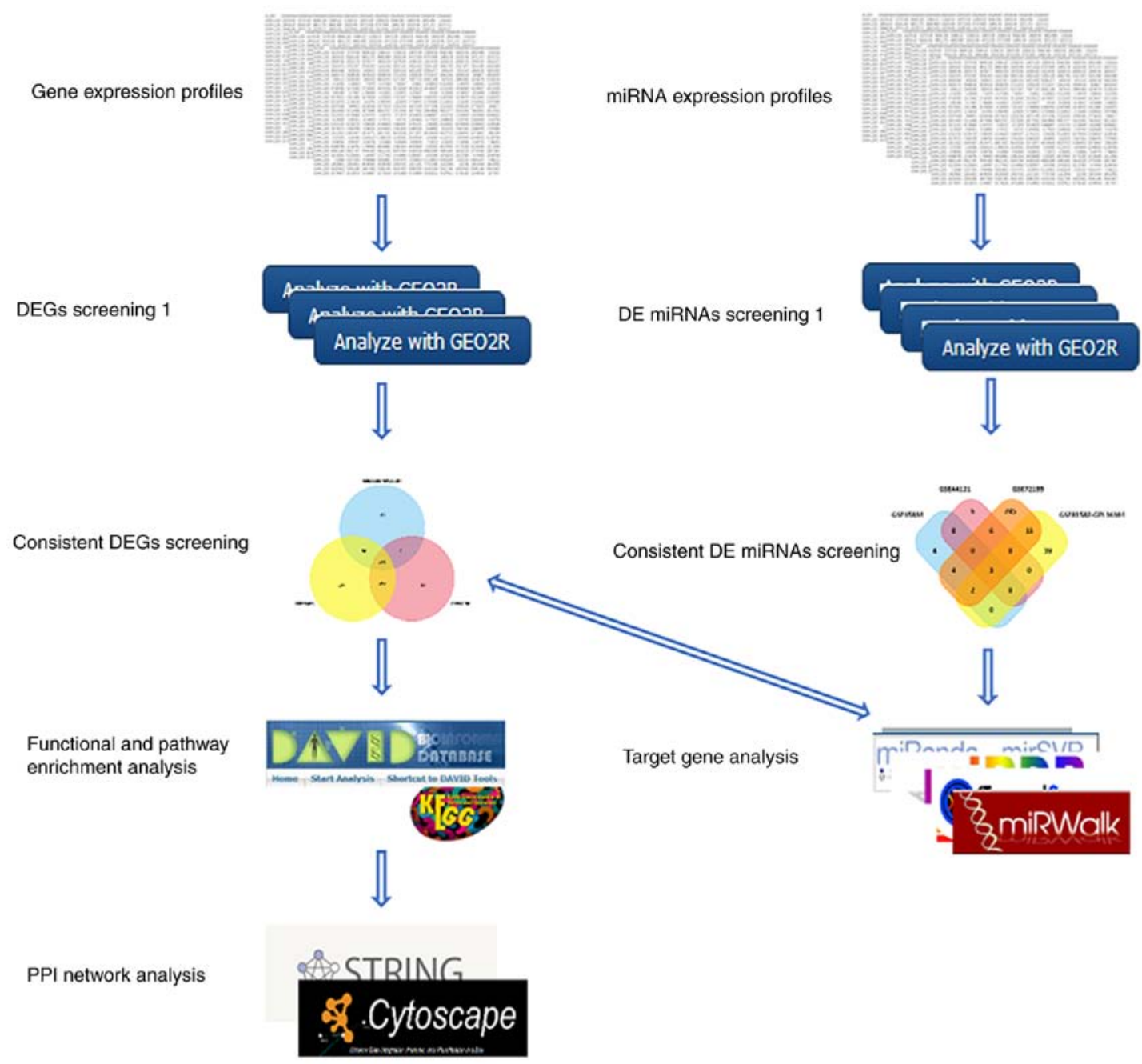

Figure 1. Flow diagram of bioinformatics analysis.

Predicting the target genes of DE miRNAs. To search for the targets of DE miRNAs, the miRanda (http://www.mocrorna. org)(23),PITA(http://genie.weizmann.ac.il/pubs/mir07/mir07_ data.html) (24), TargetScan (http://www.targetscan.org/) (25), miRDB (http://www.mirdb.org/) (26) and miRWalk (http://mirwalk.uni-hd.de/) (27) online databases were used. The genes identified in $\geq 4$ databases were considered as targets of DE miRNAs. The regulatory network between DE miRNAs and DEGs, which screened for predicted target genes, was further constructed to search for key molecules and axes in CRC liver metastasis.

Comparison of the expression level of target genes. The Gene Expression Profiling Interactive Analysis (GEPIA; http://gepia. cancer-pku.cn/index.html) is an online tool, which delivers fast and customizable analysis for RNA sequencing expression data based on The Cancer Genome Atlas and GTEx databases, which include data on 9,736 tumors and 8,587 normal samples (28). GEPIA provides key interactive and customizable functions, including differential expression analysis between cancer and normal tissues. Through its use, it could be determined whether the expression of target genes of DE miRNAs differed between CRC tissues and normal mucosa. A boxplot was constructed to visualize any altered expression.
Cell culture and transfection. The human CRC cell lines SW480 and LoVo, obtained from the Cell Bank of Chinese Academy of Sciences (Shanghai, China), were cultured in Dulbecco's modified Eagle's medium (DMEM; Gibco; Thermo Fisher Scientific, Inc., Waltham, MA, USA) with $10 \%$ fetal bovine serum (FBS) (ScienCell Research Laboratories, Inc., San Diego, CA, USA) and 1\% penicillin/streptomycin (Gibco; Thermo Fisher Scientific, Inc.). Cells were cultured at $37^{\circ} \mathrm{C}$ in an incubator with $5 \% \mathrm{CO}_{2}$.

SW480 and LoVo cells were cultured in 12-well plates and transfected with miR-885 mimics, inhibitor and miRNA negative control (NC) sequences that were purchased from Shanghai GenePharma Co., Ltd. (Shanghai, China). Transfection was conducted using Lipofectamine ${ }^{\circledR} 3000$ (Thermo Fisher Scientific, Inc.) The cells were harvested at $48 \mathrm{~h}$ after transfection for reverse transcription-quantitative polymerase chain reaction (RT-qPCR) analysis, and migration analysis.

Wound-healing assay. A scratch wound-healing assay was used to assess SW480 and LoVo cell migration. Subsequent to transfection for $48 \mathrm{~h}$, the cells were digested and a total of $3 \times 10^{5}$ cells/well were seeded into 12 well-plates and cultured overnight. Wounds were subsequently created using $10-\mu 1$ 
sterile tips. Cell migration distances were measured using an inverted microscope (Olympus Corp., Tokyo, Japan) at 0,24 and $36 \mathrm{~h}$.

Transwell migration assay. A Transwell migration assay was performed using 24 -well Transwell plates with $8-\mu \mathrm{m}$ pores (Corning Incorporated, Corning, NY, USA) to assess the migration of SW480 and LoVo cells. In total, $1 \times 10^{5}$ cells in $200 \mu 1$ FBS-free DMEM were placed in the upper Transwell chamber. Subsequently, $700 \mu 130 \%$ FBS DMEM was added to the lower chamber as a chemoattractant. Following cell culture for $24 \mathrm{~h}$, the non-invasive cells in the upper chamber were washed with cotton swabs. Migrated cells at the bottom of the membrane were fixed with $4 \%$ paraformaldehyde and stained with $0.1 \%$ crystal violet for visualization. Cells were counted in five respective fields at an $\times 200$ magnification using a light microscope (Olympus Corp.).

RNA extraction and RT-qPCR. Total RNA was extracted from cells with TRIzol ${ }^{\circledR}$ reagent (Invitrogen; Thermo Fisher Scientific, Inc.) according to the manufacturer's protocol. Subsequently, cDNA was synthesized from $2 \mu \mathrm{g}$ total RNA and quantification of miR-885 was performed using an miRNA 1st Strand cDNA Synthesis kit (by stem-loop; Vazyme Biotech Co., Ltd., Nanjing, China). Real-time PCR was performed using an Applied Biosystems StepOne Real-Time PCR system, using ChamQ ${ }^{\text {TM }}$ SYBR $^{\circledR}$ qPCR Master Mix (High ROX Premixed; Vazyme), containing 5 ng cDNA and $10 \mathrm{pM}$ of each primer. The cycling conditions consisted of 1 cycle at $95^{\circ} \mathrm{C}$ for $30 \mathrm{sec} ; 40$ cycles at $95^{\circ} \mathrm{C}$ for $10 \mathrm{sec}$; and $60^{\circ} \mathrm{C}$ for $30 \mathrm{sec}$. Melting curve analysis was conducted for each PCR reaction to confirm the specificity of amplification. The concentration of miR-885 was calculated based on the quantification cycle $(\mathrm{Cq})$, and the relative expression levels were calculated as $2^{-\Delta \Delta \mathrm{Cq}}\left[\Delta \Delta \mathrm{Cq}=\left(\mathrm{Cq}_{\mathrm{miR}-885}-\mathrm{Cq}_{\mathrm{U} 6}\right)\right.$ mimics or inhibitor - $\left.\left.\left(\mathrm{Cq}_{\mathrm{miR}-885}-\mathrm{Cq} \mathrm{q}_{\mathrm{U}}\right) \mathrm{NC}\right)\right]$ following normalization with reference to the quantification of U6 expression.

As for the target genes, RT was conducted with HiScript ${ }^{\circledR}$ II Q RT SuperMix for qPCR (+gDNA wiper; Vazyme) according to the manufacturer's protocol. The expression level of target genes was normalized to GAPDH and calculated as $2^{-\Delta \Delta C q}$. The primers wereas follows: U6 forward (F): TCGCTTCGGCAG CACATA and reverse (R): TTTGCGTGTCATCCTTGC]; miR-885 (F: GCGCGTCCATTACACTACCCT and R: AGT GCAGGGTCCGAGGTATT); IGFBP5 (F: TGAAGGCTG AAGCAGTGAAGAAGG and R: TTGTCCACGCACCAG CAGATG); vWF (F: GGCGGCAACAGGACCAACAC and R: GGAGGAGCCATCCAGGAGAAGG); and GAPDH (F: ACAACTTTGGTATCGTGGAAGG and R: GCCATCACG CCACAGTTTC).

Statistical analysis. All data were analyzed using GraphPad Prism 6.0 statistical software (GraphPad Software, Inc., La Jolla, CA, USA) and SPSS 22.0 (IBM Corp., Armonk, NY, USA). The two-tailed paired Student's t-test was conducted for the analysis of two groups. One-way analysis of variance (ANOVA) test and Bonferroni post hoc test were used for evaluating differences among multiple groups. $\mathrm{P}<0.05$ was considered to indicate a statistically significant difference.

\section{Results}

Screening for DEGs. In total, 284, 444 and 397 DEGs were extracted from the gene expression profile datasets GSE41258, GSE68468 and GSE81582-GPL15207, respectively, using $\mathrm{P}<0.05$ and $\mid \log \mathrm{FCl} \geq 1$ as cut-off criteria. Subsequently, 13, 15,776 and 59 DE miRNAs were extracted from the miRNA expression profile datasets GSE35834, GSE44121, GSE72199 and GSE81582-GPL16384, respectively. Following integrated bioinformatics analysis, a total of 141 consistent DEGs (including 97 upregulated genes and 44 downregulated genes) and 3 consistent DE miRNAs (2 upregulated and 1 downregulated) were identified from the 3 gene and 4 miRNA expression profile datasets (Fig. 2A and B), respectively, in the primary CRC tumor tissues, compared with liver metastatic CRC tumor tissues (Fig. 2C and D).

GO functional analysis and KEGG pathway enrichment analysis. DAVID was used to conduct GO functional analysis and KEGG pathway enrichment analysis. GO describes genes from three aspects, namely molecular function (MF), cellular component (CC) and biological process (BP) (29). In the BP group, upregulated DEGs were primarily enriched in 'acute-phase response', 'platelet degranulation', 'negative regulation of endopeptidase activity', 'fibrinolysis', 'negative regulation of fibrinolysis', 'lipoprotein metabolic process', 'retinoid metabolic process', 'steroid metabolic process', 'cholesterol efflux' and 'blood coagulation' (Fig. 3A). Downregulated DEGs were primarily enriched in 'collagen catabolic process', 'proteolysis', 'extracellular matrix disassembly', 'positive regulation of B cell activation', 'phagocytosis, recognition', 'phagocytosis, engulfment', 'negative regulation of BMP signaling pathway', 'B cell receptor signaling pathway', 'extracellular matrix organization' and 'complement activation, classical pathway' (Fig. 4A).

In the CC group, upregulated DEGs were primarily enriched in 'blood microparticle', 'extracellular region', 'extracellular space' and 'extracellular exosome' (Fig. 3B). Downregulated DEGs were primarily enriched in the 'extracellular region', 'proteinaceous extracellular matrix', 'extracellular space', 'extracellular exosome' and 'blood microparticle' (Fig. 4B).

In the MF group, upregulated DEGs were primarily enriched in 'serine-type endopeptidase inhibitor activity', 'lipase inhibitor activity', 'heparin binding', 'endopeptidase inhibitor activity', 'receptor binding', 'phosphatidylcholine-sterol O-acyltransferase activator activity', 'lipid transporter activity', 'phospholipid binding', 'monooxygenase activity' and 'cholesterol transporter activity' (Fig. 3C). Downregulated DEGs were primarily enriched in 'serine-type endopeptidase activity', 'immunoglobulin receptor binding', 'collagen binding', 'fibronectin binding', 'antigen binding', 'extracellular matrix structural constituent', 'peptidase activator activity', 'metalloendopeptidase activity' and 'receptor agonist activity' (Fig. 4C).

In the KEGG pathway enrichment analysis, upregulated DEGs were primarily enriched in 'complement and coagulation cascades', 'drug metabolism-cytochrome P450', 'metabolism of xenobiotics by cytochrome P450', 'chemical carcinogenesis', 'retinol metabolism', 'steroid hormone biosynthesis', 'tyrosine 
A

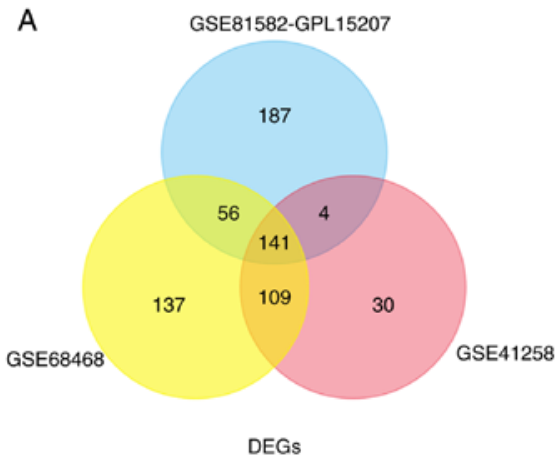

C

\begin{tabular}{|c|c|}
\hline Regulation & DEGs \\
\hline $\begin{array}{l}\text { Upregulated } \\
\qquad(n=97)\end{array}$ & $\begin{array}{l}\text { AADAC, ADH1A, ADJH1B, AGXT, AHSG, AKR1C1, AKR1C2, ALB, } \\
\text { ALDH8A1, ALDOB, AMBP, AOX1, APCS, APOA1, APOA2, APOB, } \\
\text { APOC1, APOC2, APOC3, APOC4, APOE, APOH, ARG1, ASGR1, } \\
\text { AZGP1, BAAT, C4BPA, C5, C8A, C9, CDH2, CFH, CFHR1, CFHR2, } \\
\text { CFHR4, CFHR5, COLEC11, CP, CPB2, CPS1, CRP, CYP2C8, CYP2 } \\
\text { CYP2E1, CYP3A4, F2, F5, F9, FGA, FGB, FGG, FGL1, FMO3, G6P } \\
\text { GATM, GC, HGD, HP, HPD, HPR, HPX, HRG, IGFBP1, ITIH2, ITIH3, } \\
\text { ITIH4, KNG1, LBP, LECT2, ;LEPR, MBL2, ORM1, ORM2, PAH, PLG, } \\
\text { PLGLB1, PLGLB2, RBP4, RGN, SAA1, SAA2, SERPINA1, } \\
\text { SERPINA10, SERPINA3, SERPINC1, SERPIND1, SERPINF2, SLC2 } \\
\text { SLC01B3, SPP1, SULT2A1, TF, TTR, UGT2B15, UGT2B4, VNN1, VT }\end{array}$ \\
\hline $\begin{array}{l}\text { Downregulated } \\
\qquad(\mathrm{n}=44)\end{array}$ & $\begin{array}{l}\text { ACTG2, ALDH1A3, ANGPTL2, ANTXR1, CAV1, CDH11, CHL1, } \\
\text { CHN1, CHRDL1, CNN1, COL11A1, CTSK, CXCL14, DES, DIO2, } \\
\text { DKK3, FBLN1, FCGBP, FHL1, FOXF1, GAS1, GREM1, IGFBP5, } \\
\text { IGHG1. IGHG3, IGHM, IGHV4-31, IGKC, MFAP5, MMP1, MMP2, } \\
\text { MMP3, MYH11, PDZD2. PLN, RGS5, SPARCL1, SPINK4, SPON1, } \\
\text { TMEM158, TPSAB1, TPSB2, VWF, WNT5A }\end{array}$ \\
\hline \multicolumn{2}{|c|}{ Regulation DE miRNAs } \\
\hline \multicolumn{2}{|l|}{ Upregulated $(n=2)$} \\
\hline Downregulated $(\mathrm{n}=$ & miR-10b \\
\hline
\end{tabular}

B

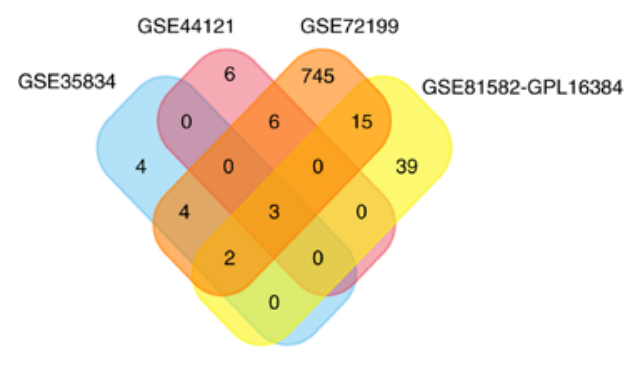

DE MiRNAS

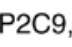

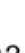


A

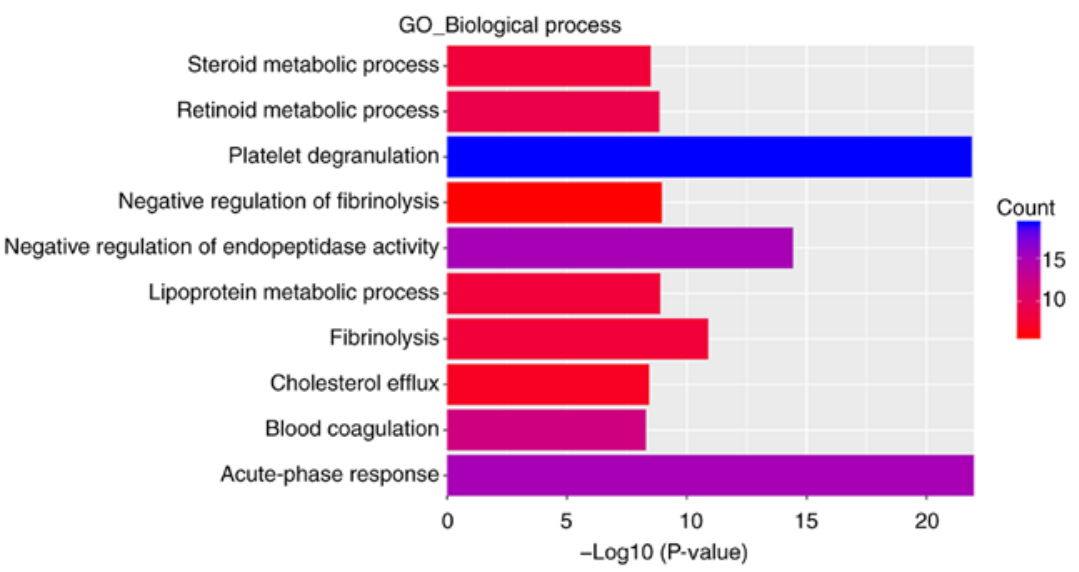

B

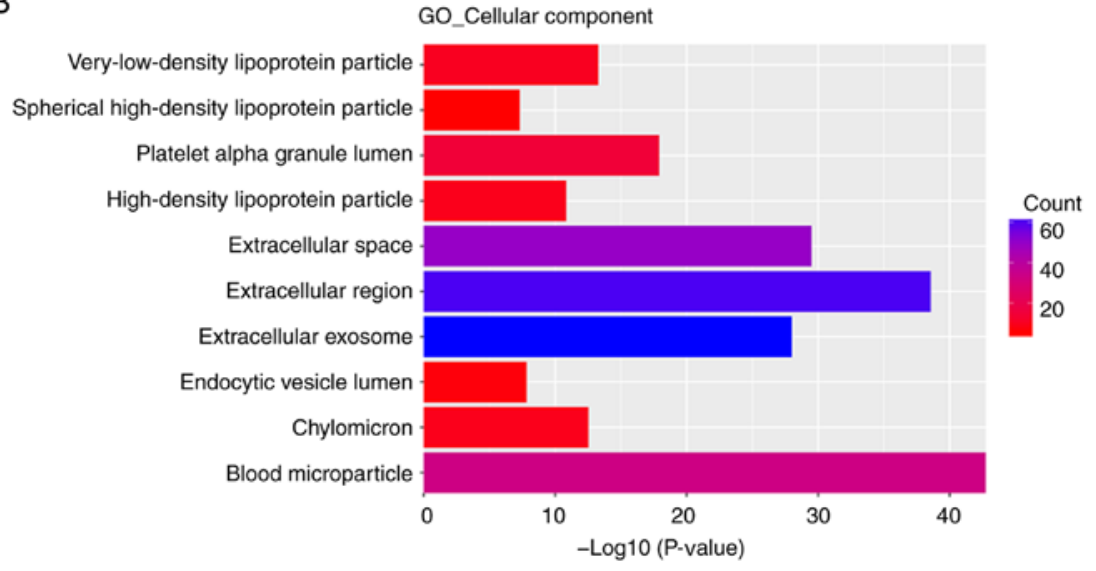

C

GO_Molecular function

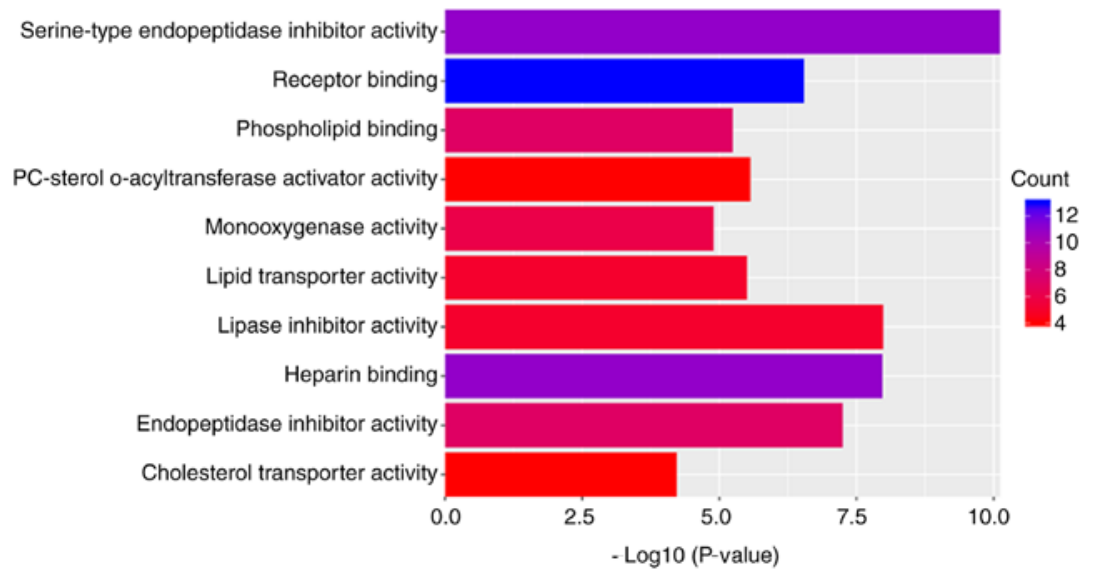

D

KEGG pathway

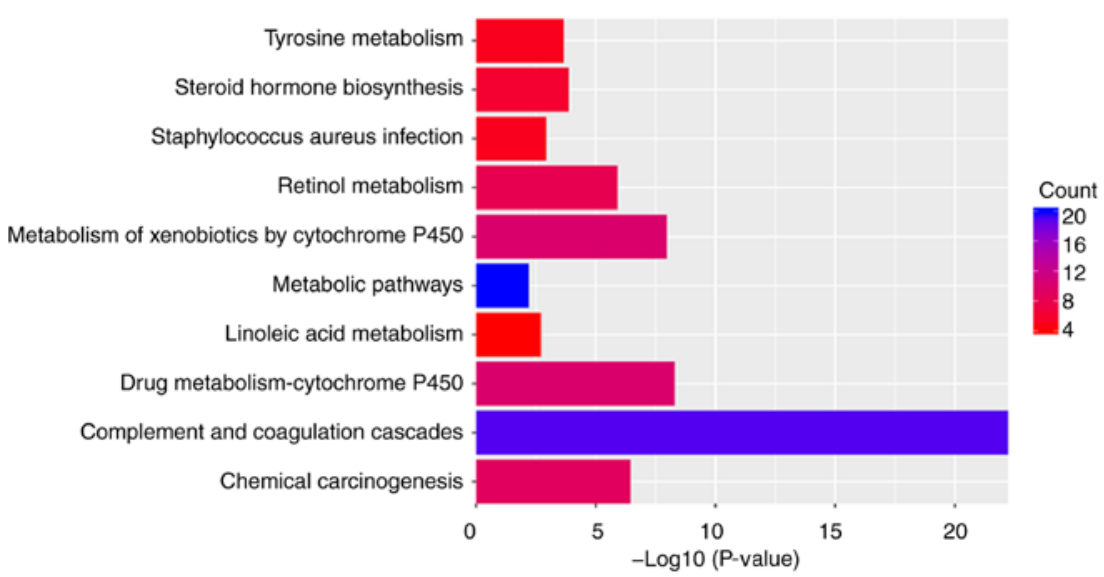

Figure 3. GO and KEGG pathway enrichment analysis of upregulated DEGs. (A) Biological process. (B) Cellular component. (C) Molecular function. (D) KEGG pathway enrichment analysis. GO, Gene Ontology; KEGG, Kyoto Encyclopedia of Genes and Genomes; DEGs, differentially expressed genes. 
A

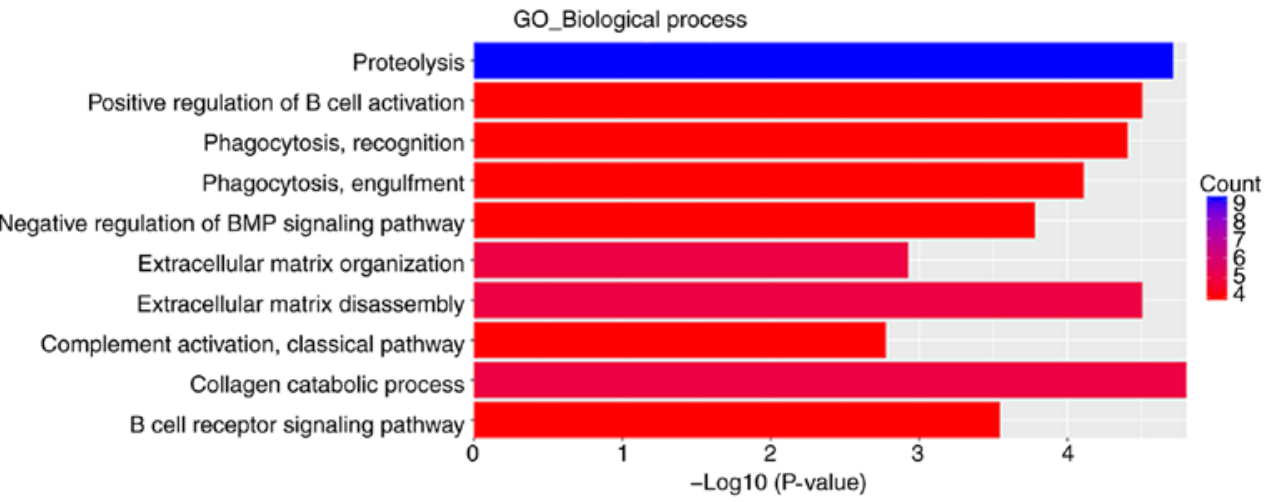

B

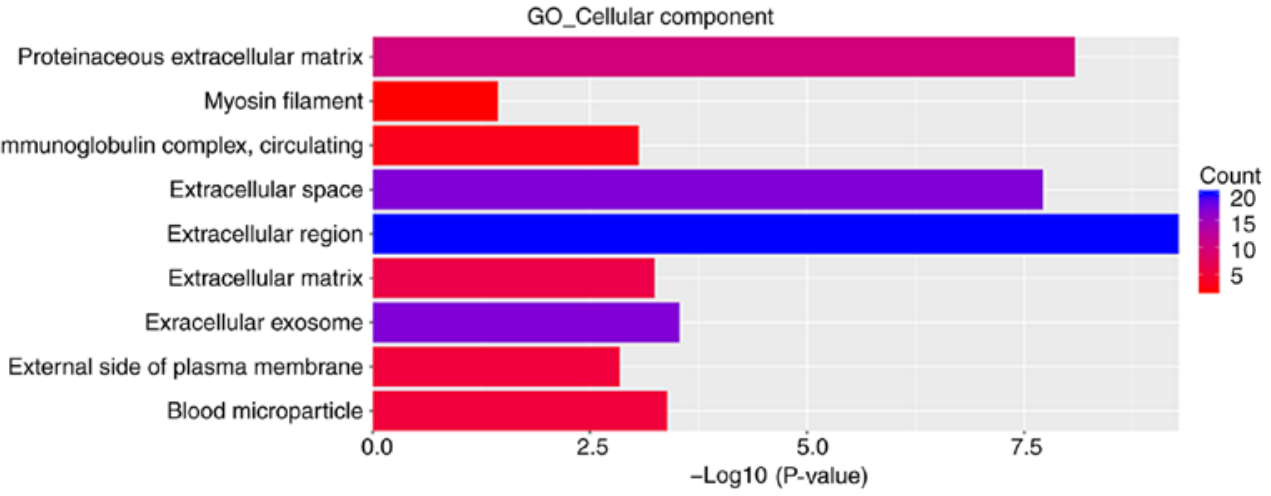

C

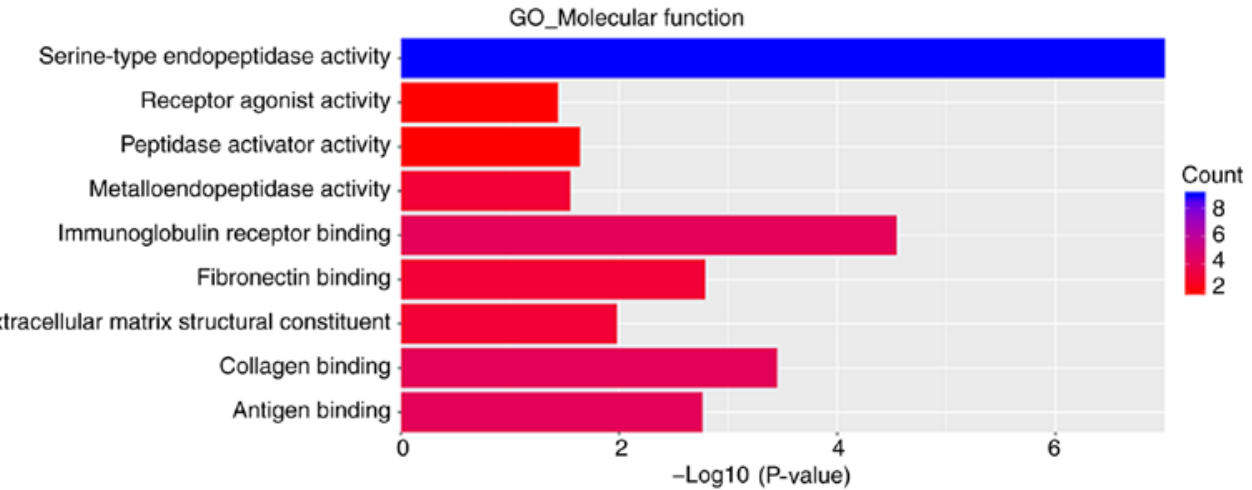

Figure 4. GO enrichment analysis of downregulated DEGs. (A) Biological process. (B) Cellular component. (C) Molecular function. GO, Gene Ontology; DEGs, differentially expressed genes.

and these target DEGs in CRC, further analysis of the expression level of DE miRNAs in CRC tumor tissues and normal mucosa in GSE35834 and GSE81582-GPL16384 was conducted. Similar significant expression differences of these DE miRNAs were identified between CRC tumor and normal mucosa (Fig. 7A and B). The GEPIA database was used to compare the expression level of these genes between normal mucosa and CRC tissues. Among the 20 target DEGs, consistent with the downregulation in liver metastases, the expression of IGFBP5, microfibril associated protein 5 (MFAP5), myosin heavy chain 11 (MYH11) and vWF were significantly downregulated $(\mathrm{P}<0.05$; $\mid \log \mathrm{FCl} \geq 1)$ in $\mathrm{CRC}$ tissues compared with normal mucosa (Fig. 7C).

miR-885 promotes CRC cell migration in vitro. The role of miR-122 and miR-10b in CRC has been widely investigated; however, few previous studies have focused on the role of miR-885 in CRC. To evaluate the possible functions of miR-885 in CRC progression, miR-885 mimics and inhibitor were transfected into SW480 and LoVo cells to obtain cells with overexpression and low expression of miR-885 (Fig. 8A and B). The effects of miR-885 on the migration of CRC cells were observed by Transwell migration assays and wound healing assays. It was revealed that overexpression of miR-885 promoted the migration abilities of the CRC cells in vitro. Additionally, the inhibition of miR-885 suppressed the migration abilities of CRC (Fig. 8C-F).

miR-885 decreases $v W F$ and IGFBP5 expression in CRC. In order to verify the regulatory networks of $\mathrm{miR}-885 / \mathrm{vWF}$ and miR-885/IGFBP5, PCR analysis was performed. The results demonstrated that the vWF and IGFBP5 mRNA expression levels were significantly downregulated in miR-885-overexpressing cells; whereas, these expression levels were upregulated in the cells with low expression of miR-885 (Fig. 9). 

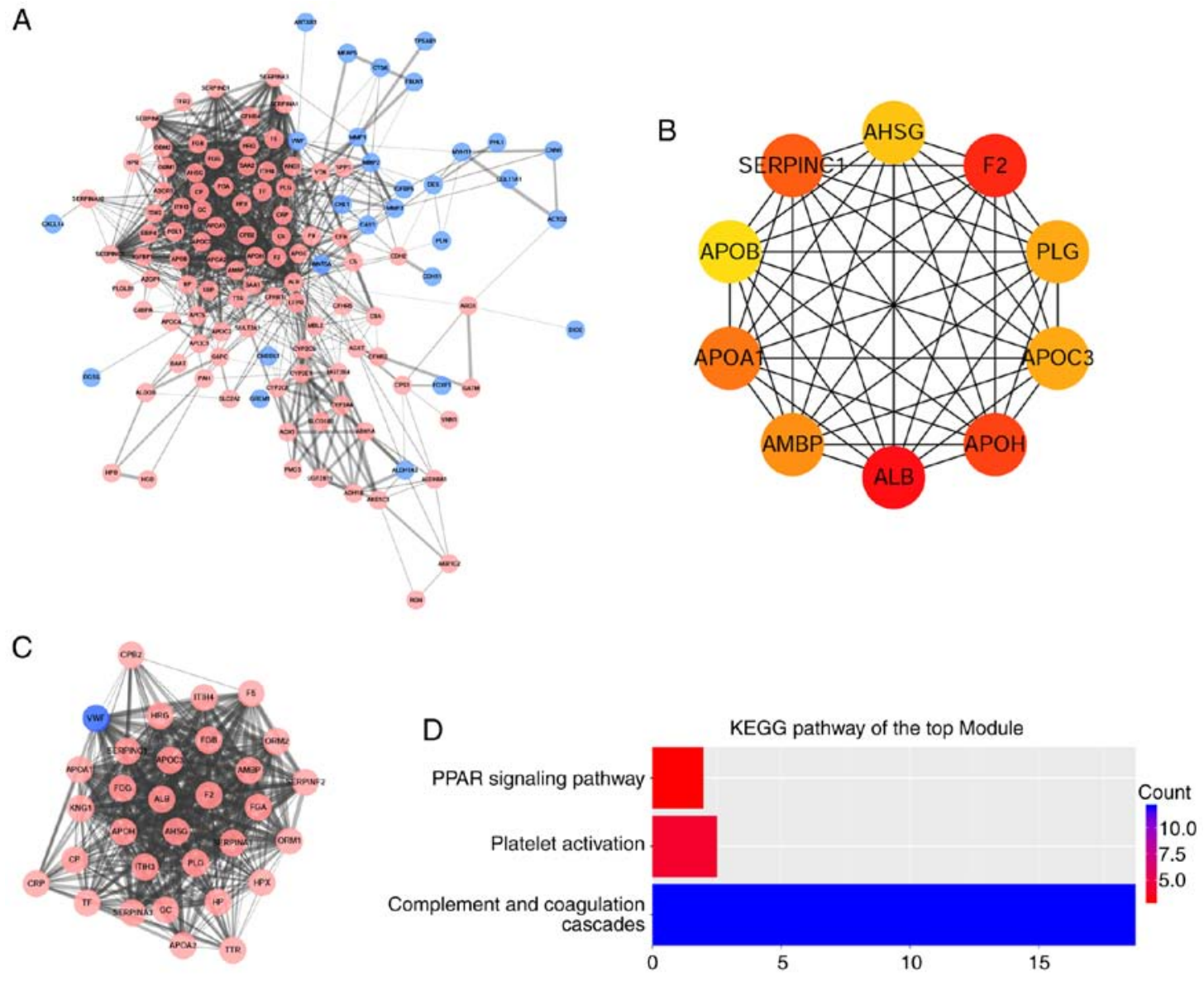

Figure 5. PPI network and module analysis. (A) PPI network of DEGs. The pink nodes indicated upregulated genes and the blue nodes indicated downregulated genes. The edge thickness was proportional to the combined score of the genes. (B) Top 10 hub genes with a higher degree of connectivity. (C) Top module screened from the PPI network. (D) KEGG pathway enrichment analysis of the top module. PPI, protein-protein interaction; DEGs, differentially expressed genes; KEGG, Kyoto Encyclopedia of Genes and Genomes.

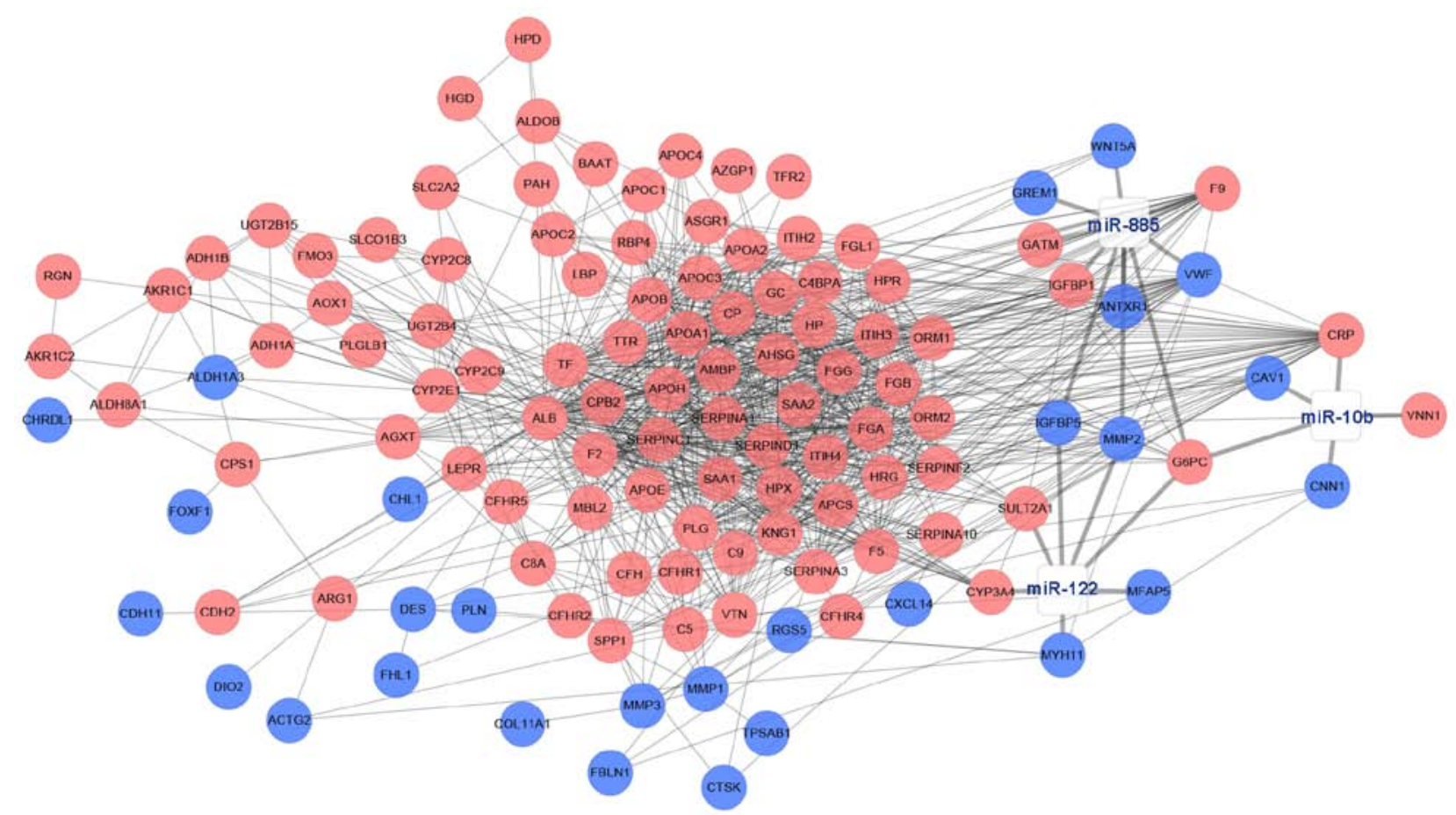

Figure 6. DE miRNAs-DEGs regulatory network. The pink nodes indicated upregulated DEGs, the blue nodes indicated downregulated DEGs, and the white nodes indicated DE miRNAs. The thick lines indicated the regulation relationship between DE miRNAs and DEGs, while the thin lines indicated the relationship between DEGs. DE, differentially expressed; miRNAs, microRNAs; DEGs, differentially expressed genes. 
A
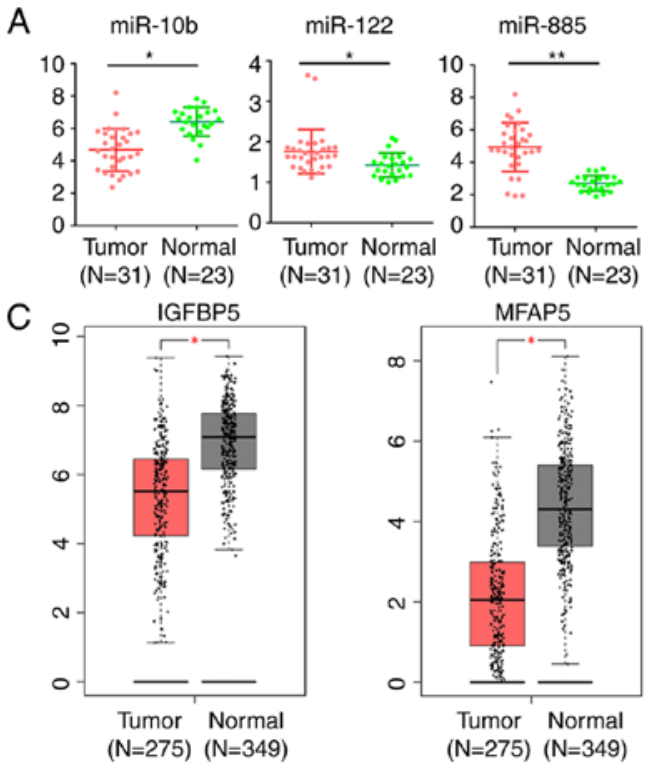

MFAP5

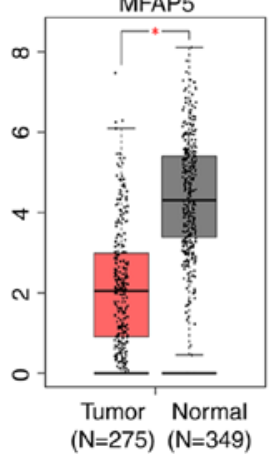

B
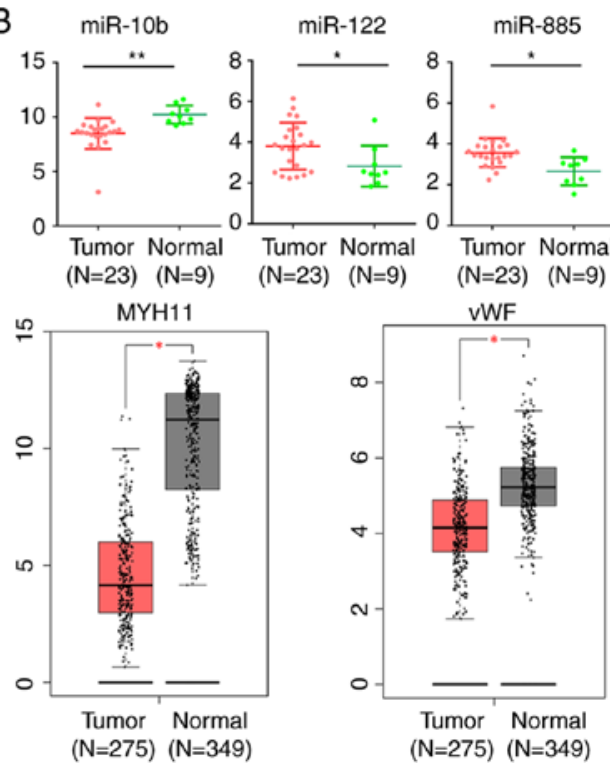

Figure 7. Expression level of DE miRs and target DEGs in CRC and normal mucosa. Expression level of miR-10b, miR-122 and miR-885 in CRC and normal mucosa in (A) GSE35834 and (B) GSE81582-GPL16384. ${ }^{*} \mathrm{P}<0.05,{ }^{* *} \mathrm{P}<0.01$. (C) The expression level of IGFBP5, MFAP5, MYH11 and vWF in CRC and normal mucosa based on TCGA database analyzed by GEPIA. The red plot represents tumor tissues and the grey plot represents normal mucosa. * $\mathrm{P}<0.05$ and $\mid \log \mathrm{FCl} \geq 1$ were set as the criteria. DE, differentially expressed; miR, microRNA; DEGs, differentially expressed genes; CRC, colorectal cancer; IGFBP5, insulin-like growth factor binding protein 5; MFAP5, microfibril associated protein 5; MYH11, myosin heavy chain 11; TCGA, The Cancer Genome Atlas; FC, fold change; vWF, von Willebrand factor; GEIPA, Gene Expression Profiling Interactive Analysis.

A

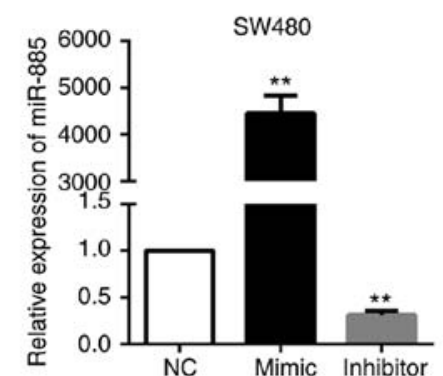

C

SW480

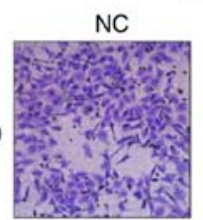

LoVo

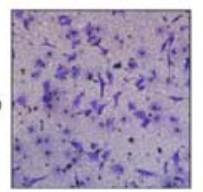

E

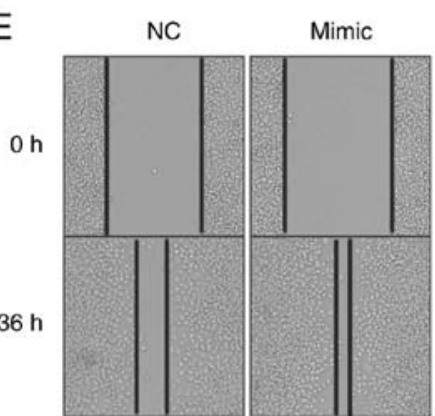

SW480
B
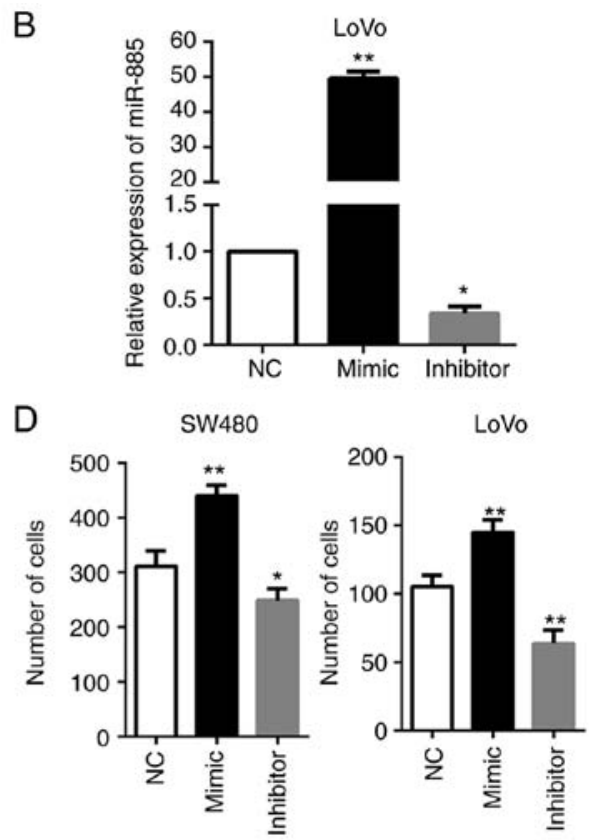

F

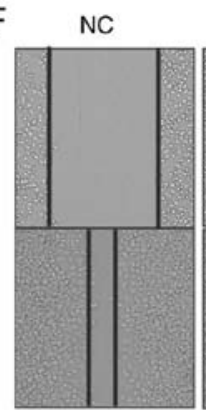

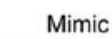

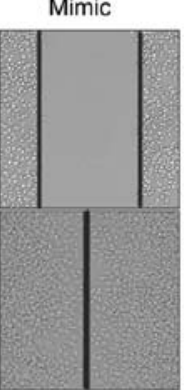

LoVo

Figure 8. miR-885 promotes the migration of CRC cells in vitro. (A and B) Expression of miR-885 in SW480 and LoVo cells transfected with inhibitor, mimics or the negative control was detected by polymerase chain reaction assays. (C and D) Transwell migration assays. Representative images and quantification of Transwell migration assays of SW480 and LoVo cells following miR-885 overexpression and knockdown. (E and F) Wound-healing assay of SW480 and LoVo cells at 0 and $36 \mathrm{~h}$. Error bars represent the mean \pm standard deviation from three independent experiments. ${ }^{*} \mathrm{P}<0.05,{ }^{* *} \mathrm{P}<0.01$. miR, microRNA; CRC, colorectal cancer. 

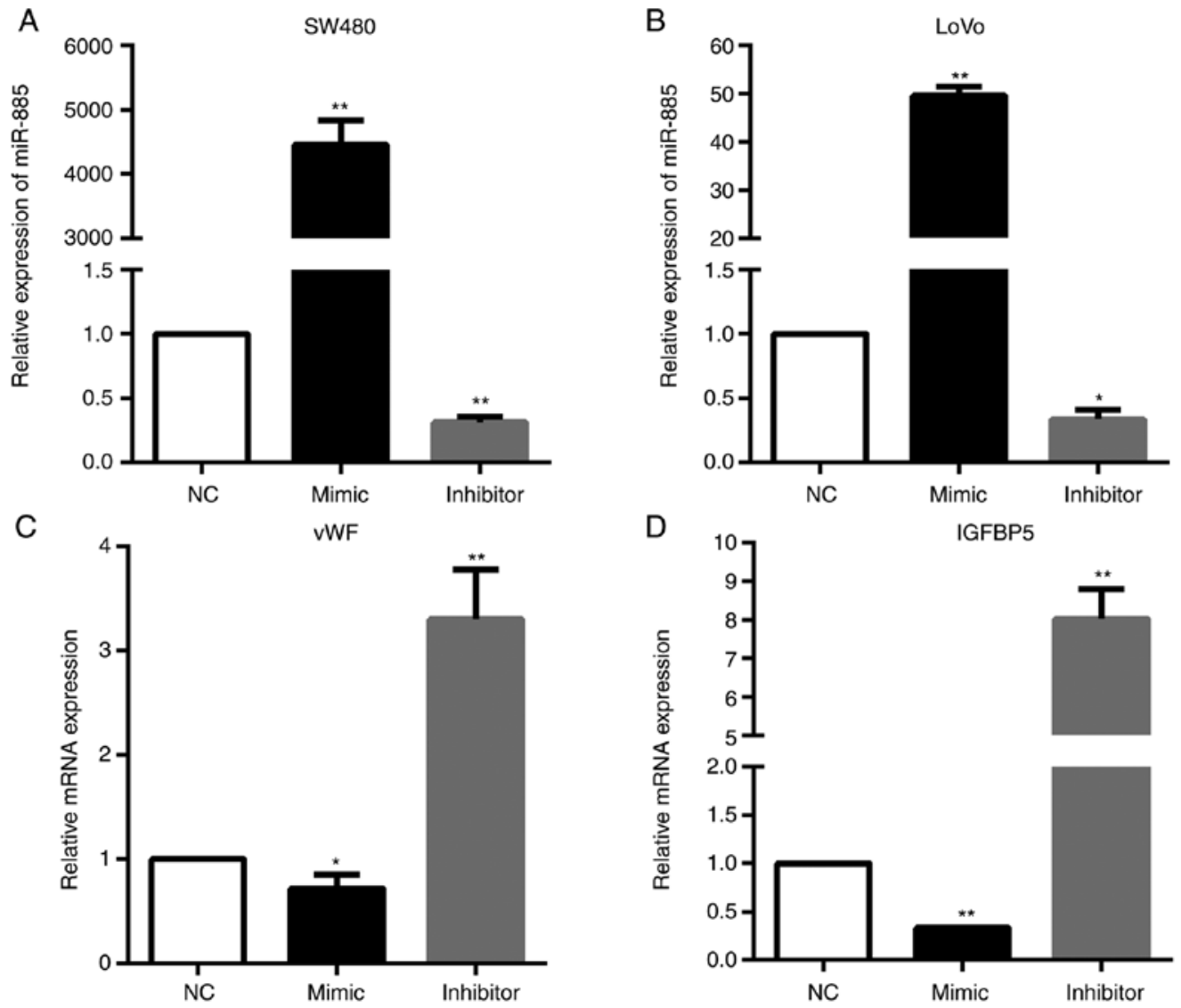

Figure 9. miR-885 decreases vWF and IGFBP5 expression. (A and B) Expression of miR-885 in SW480 and LoVo cells transfected with inhibitor, mimics or the negative control was detected by PCR. (C and D) PCR analysis of vWF and IGFBP5 mRNA expression in the LoVo cells. Error bars represent the mean \pm standard deviation from three independent experiments. ${ }^{*} \mathrm{P}<0.05,{ }^{* *} \mathrm{P}<0.01$. miR, microRNA; vWF, von Willebrand factor; IGFBP5, insulin-like growth factor binding protein 5; PCR, polymerase chain reaction.

\section{Discussion}

A number of pre-clinical and clinical studies have been conducted to reveal the underlying mechanisms of CRC liver metastasis in the past decades; however, the incidence and mortality of CRC liver metastasis remain high. This is primarily due to the majority of the studies focusing on a single genetic event, or the results were generated from a single cohort study (30). In the present study, 3 gene expression profile datasets were integrated and 141 commonly altered DEGs (97 upregulated and 44 downregulated) were identified. The 141 DEGs were classified into three groups (BP, CC and MF groups) by GO terms, and the KEGG pathway enrichment analysis of the DEGs was conducted using the DAVID database. Finally, the DEGs PPI network was constructed, and the top 10 hub genes and the most significant module was filtered from the PPI network.

Through integrated bioinformatics analysis, 10 hub genes were identified, including ALB, F2, APOH, SERPINC1, APOA1, AMBP, APOC3, PLG, AHSG and APOB with a high degree of connectivity. Among them, APOH, APOA1, APOC 3 and APOB belong to the apolipoprotein family, and their biological functions are primarily involved in the lipoprotein metabolism process and cholesterol transport (31). Multiple previous studies have demonstrated that apolipoproteins additionally serve a role in the occurrence and development of varies malignancies (32). APOA1 is the principal protein constituent of high-density lipoprotein that interact with ATP-binding cassette subfamily A member 1, ATP-binding cassette subfamily $\mathrm{G}$ member 1 and lecithin cholesterol acyltransferase to promote cholesterol efflux from cells to the liver for excretion (33). By eliminating excessive cholesterol, APOA1 exerts anti-inflammatory, anti-apoptotic and antioxidant activities, thus serving a protective role against cancer; chronic inflammation, oxidative stress, lipids and cholesterol have all been associated with tumorigenesis (33). In addition, APOA1 suppresses tumor growth by inhibiting myeloid-derived suppressor cells and their immuno-suppression for tumor growth, invasion and metastasis, by decreasing MMP-9 expression (34). A recent study demonstrated that serum APOA1 was significantly decreased in patients with CRC (35), and that a low serum APOA1 expression level was associated with poor survival and advanced stage in CRC (36). However, the present bioinformatics analysis demonstrated a significant increase in APOA1 expression level in liver metastatic CRC tissues, indicating a stimulatory effect of APOA1 in CRC metastasis. The role of APOB, APOC3 and $\mathrm{APOH}$ in the tumorigenesis and metastasis of CRC remains unclear. However, a high APOB expression level was associated with increased lung cancer incidence (32), and the APOB-100 gene polymorphisms may be associated with increased risk of gallstones and gallbladder cancer (37). APOC3 was overexpressed in multiple hepatocellular carcinoma (HCC) family case studies and may be involved in $\mathrm{HCC}$ familial aggregation (38). APOH, 
additionally termed $\beta 2$ glycoprotein I, was observed to affect endothelial cell growth and angiogenesis (39), and serve roles in lipopolysaccharide-induced signal transduction involved in promoting the development of HCC (40). Therefore, further pre-clinical and clinical studies are required to determine the exact role and mechanisms of these apolipoproteins in CRC liver metastasis.

Furthermore, other hub genes may additionally be involved in the metastasis of CRC. AMBP is a glycoprotein that is normally highly expressed in the liver and may be detected in plasma and urine (41). AMBP is able to hydrolyze into two distinct functional proteins, $\alpha-1$-microglobulin and bikunin, which are involved in multiple biological processes, including cell growth, cellular calcium uptake and inflammation (41). At present, there is no study, to the best of our knowledge, examining the role of AMBP in CRC. However, it was documented that AMBP was overexpressed in patients with gastric cancer, and a high expression level of serum AMBP was associated with poor response to paclitaxel-capecitabine chemotherapy in patients with advanced gastric cancer (41). AHSG is a negative acute phase protein in humans, primarily produced by the liver. Accumulating evidence suggests that it is a multifunctional protein capable of modulating the etiology of diabetes and other metabolic diseases (42), and promoting the invasion of tumor cells (43). Furthermore, upregulated AHSG was determined in CRC, which may be used as a diagnostic marker for CRC (44). The PLG system serves a crucial role in the process of immune response, angiogenesis, invasion and metastasis, which are crucial for cancer progression (45). A previous study demonstrated that PLG deficiency markedly reduced the number of pulmonary metastases in a mouse breast cancer model, indicating the stimulatory role of PLG in tumor metastasis (46).

Additionally, the most significant module was filtered from the PPI network, among which the majority of the corresponding genes were mostly associated with complement and coagulation cascades. It was demonstrated that cancer increases the risk of thrombosis by a 4.1-fold (47), and results in the hyperactivation of coagulation and clotting abnormalities in cancer, referred to as Trousseau's syndrome. Additional symptoms include venous thromboembolism, deep vein thrombosis, disseminated intravascular coagulation and pulmonary embolism (48). Recent evidence has demonstrated that hypercoagulation and activation of complement cascades promote the pro-tumorigenic phenotype of immune cells and protect tumor cells from immune attack, ultimately favoring tumor development, progression and metastases formation (49).

Tumor-promoting inflammation serves an important role in carcinogenesis and cancer progression. As an important component of tumor-promoting inflammation, activation of the complement system promotes cancer cell proliferation, dedifferentiation and migration (50). Complement activation regulates the adaptive immune response and may play a role in regulating the $\mathrm{T}$-cell response to tumors (50). In addition, specific experimental and clinical evidence suggests a reciprocal interaction between complement and coagulation. Complement may induce hyperactivation of the coagulation cascade by modifying cellular membranes, inducing platelet activation and aggregation, and stimulating the production of tissue factor in human neutrophils (49).
The formation of CRC metastases in liver requires primary CRC cells to adapt to growing in a different and harsh environment. This adaptation requires multiple alterations in gene expression, which may be translationally and post-transcriptionally regulated through the up- or downregulation of multiple miRNAs (51). Therefore, 3 gene expression profile datasets were integrated, common DEGs were identified and bioinformatics methods were used to analyze these datasets thoroughly. Furthermore, 3 miRNA expression profile datasets were integrated and 3 common DE miRNAs (two upregulated miRNAs, miR-122 and miR-885, and one downregulated miRNA, miR-10b) were identified. Additionally, similarly significant expression differences of these DE miRNAs were additionally observed between CRC tumor and normal mucosa, suggesting an oncogenetic or tumor suppressive role of these DE miRNAs. In agreement with the present study, recent studies observed an upregulation of miR-885 and miR-122, and a downregulation of miR-10b in liver metastatic CRC tissues, suggesting that they serve as metastasis-specific miRNAs $(52,53)$. In addition, high miR-885 expression was statistically significantly associated with poor prognosis in patients with CRC, and serum miR-885 expression was statistically significantly associated with lymph node metastasis, distant metastasis, tumor, node, metastasis (TNM) stage, liver metastasis and lymphatic invasion (52). A recent study demonstrated that miR-885 serves a crucial role in liver metastasis by enhancing cell invasiveness, and regulating the epithelial-mesenchymal transition pathway by silencing CPEB2 (17). However, in contrast to the consistently decreased expression level in liver metastatic CRC tissues in different previous studies, the expression of miR-10b is paradoxical in CRC tissues compared with normal adjacent mucosa $(12,54)$, and high miR-10b expression was statistically significantly associated with poor prognosis in patients with CRC $(12,54)$. Additionally, increased miR-10b expression was statistically significantly different among $\mathrm{T}$ stage, distant metastasis and advanced TNM stage $(52,55)$. Therefore, whether miR-10b is an oncogenic miRNA or a tumor suppressor in $\mathrm{CRC}$ requires further investigation.

Additionally, to unveil a comprehensive regulatory network and screen out key molecules and axes in CRC liver metastasis, a DE miRNA-DEGs regulatory network was constructed, in which, the miR-122/IGFBP5, miR-122/MFAP5, miR-122/MYH11, miR-885/IGFBP5 and miR-885/vWF axes may serve critical roles in the occurrence and metastatic process of CRC. IGFBP5 is a member of the family of insulin-like growth factor-binding proteins, which has been demonstrated to regulate cell growth, differentiation, apoptosis and modulate the metastatic process in the development of multiple cancer types (56). However, the expression of IGFBP5 varies from different types of cancer and it serves as either an oncogene or tumor suppressor in different tumors (56). Unlike the present predictions, a previous study demonstrated that IGFBP5 was upregulated in CRC (57) and promoted CRC metastasis (58). $\mathrm{vWF}$ is a protein that modulates adherence of platelets to the subendothelium during primary hemostasis, which is crucial in the initiation of the hemostatic or thrombotic process (59). vWF serves pro- and antitumor roles in different cancer types (60). Clinical studies demonstrated that high vWF plasma expression levels were associated with advanced tumor stage 
and the presence of distant metastasis in CRC (61). However, the cleavage of vWF by a disintegrin and metalloproteinase 28 enhanced tumor metastasis, indicating an anti-metastatic role of vWF (62). The present experiments demonstrated that miR-885 promotes CRC migration, which may decrease vWF and IGFBP5 expression, at least partially, indicating that the miR-885/vWF and miR-885/IGFBP5 axes may serve roles in the metastasis of CRC. However, the present results require in vivo and further experiments, including luciferase reporter assays, for confirmation.

In summary, the present bioinformatics analysis identified 10 hub genes, including ALB, F2, APOH, SERPINC1, APOA1, APOC 3 , AMBP, PLG, AHSG and APOB, and 3 DE miRNAs, namely miR-10b, miR-122 and miR-885. The hub genes and DE miRNAs may be used as novel biomarkers for predicting the liver metastasis of CRC. Additionally, a DE miRNA-DEGs regulatory network was constructed, which may help to elucidate the underlying molecular mechanisms of liver metastasis of CRC. Furthermore, the present experiments demonstrated that miR-885 promoted CRC cell migration by, at least partially, decreasing vWF and IGFBP5 expression. In order to obtain more accurate correlation results, a large number of clinical samples and further experiments are required to validate the present results and elucidate the underlying mechanisms of how these key genes and miRNAs impact liver metastasis of CRC. The present study may provide insight for future diagnosis and genomic therapy for liver metastatic CRC.

\section{Acknowledgements}

Not applicable.

\section{Funding}

The present study was supported by the Natural Science Foundation of China (grant no. 81570568).

\section{Availability of data and materials}

The datasets used during the present study are available from the corresponding author upon reasonable request.

\section{Authors' contributions}

TZ, JGuo, JW and HL conceived and designed the study. TZ, JGuo, GW and JGu performed the data acquisition and analysis. JGu and $\mathrm{ZW}$ performed the experiments. TZ and JGuo wrote the paper. HL, JW, ZW and GW reviewed and edited the manuscript. All authors read and approved the manuscript and agree to be accountable for all aspects of the research in ensuring that the accuracy or integrity of any part of the work is appropriately investigated and resolved.

\section{Ethics approval and consent to participate}

Not applicable.

\section{Patient consent for publication}

Not applicable.

\section{Competing interests}

The authors declare that they have no competing interests.

\section{References}

1. Ferlay J, Soerjomataram I, Dikshit R, Eser S, Mathers C, Rebelo M, Parkin DM, Forman D and Bray F: Cancer incidence and mortality worldwide: Sources, methods and major patterns in GLOBOCAN 2012. Int J Cancer 136: E359-E386, 2015.

2. Siegel RL, Miller KD and Jemal A: Cancer statistics, 2017. CA Cancer J Clin 67: 7-30, 2017.

3. Chen W, Zheng R, Baade PD, Zhang S, Zeng H, Bray F, Jemal A, $\mathrm{Yu}$ XQ and He J: Cancer statistics in China, 2015. CA Cancer J Clin 66: 115-132, 2016.

4. Manfredi S, Lepage C, Hatem C, Coatmeur O, Faivre J and Bouvier AM: Epidemiology and management of liver metastases from colorectal cancer. Ann Surg 244: 254-259, 2006.

5. Bakalakos EA, Kim JA, Young DC and Martin EW Jr: Determinants of survival following hepatic resection for metastatic colorectal cancer. World J Surg 22: 399-404; discussion 404-395, 1998.

6. Bartel DP: MicroRNAs: Target recognition and regulatory functions. Cell 136: 215-233, 2009.

7. Hur K: MicroRNAs: Promising biomarkers for diagnosis and therapeutic targets in human colorectal cancer metastasis. BMB Rep 48: 217-222, 2015.

8. Kulasingam V and Diamandis EP: Strategies for discovering novel cancer biomarkers through utilization of emerging technologies. Nat Clin Pract Oncol 5: 588-599, 2008.

9. Sheffer M, Bacolod MD, Zuk O, Giardina SF, Pincas H, Barany F, Paty PB, Gerald WL, Notterman DA and Domany E: Association of survival and disease progression with chromosomal instability: A genomic exploration of colorectal cancer. Proc Natl Acad Sci USA 106: 7131-7136, 2009.

10. Tsafrir D, Tsafrir I, Ein-Dor L, Zuk O, Notterman DA and Domany E: Sorting points into neighborhoods (SPIN): Data analysis and visualization by ordering distance matrices. Bioinformatics 21: 2301-2308, 2005.

11. Sayagues JM, Corchete LA, Gutierrez ML, Sarasquete ME, Del Mar Abad M, Bengoechea O, Fermiñán E, Anduaga MF, Del Carmen S, Iglesias M, et al: Genomic characterization of liver metastases from colorectal cancer patients. Oncotarget 7 : 72908-72922, 2016.

12. Pizzini S, Bisognin A, Mandruzzato S, Biasiolo M, Facciolli A, Perilli L, Rossi E, Esposito G, Rugge M, Pilati P, et al: Impact of microRNAs on regulatory networks and pathways in human colorectal carcinogenesis and development of metastasis. BMC genomics 14: 589, 2013.

13. Mokutani Y, Uemura M, Munakata K, Okuzaki D, Haraguchi N, Takahashi H, Nishimura J, Hata T, Murata K, Takemasa I, et al: Down-regulation of microRNA-132 is associated with poor prognosis of colorectal cancer. Ann Surg Oncol 23: 599-608, 2016.

14. Yan W, Zhang W, Sun L, Liu Y, You G, Wang Y, Kang C, You Y and Jiang T: Identification of MMP-9 specific microRNA expression profile as potential targets of anti-invasion therapy in glioblastoma multiforme. Brain Res 1411: 108-115, 2011.

15. Afanasyeva EA, Mestdagh P, Kumps C, Vandesompele J, Ehemann V, Theissen J, Fischer M, Zapatka M, Brors B, Savelyeva L, et al: MicroRNA miR-885-5p targets CDK2 and MCM5, activates p53 and inhibits proliferation and survival. Cell Death Differ 18: 974-984, 2011.

16. Zhang Z, Yin J, Yang J, Shen W, Zhang C, Mou W, Luo J, Yan H, Sun P, Luo Y, et al: miR-885-5p suppresses hepatocellular carcinoma metastasis and inhibits Wnt/beta-catenin signaling pathway. Oncotarget 7: 75038-75051, 2016.

17. Lam CS, Ng L, Chow AK, Wan TM, Yau S, Cheng NS, Wong SK, Man JH, Lo OS, Foo DC, et al: Identification of microRNA $885-5 \mathrm{p}$ as a novel regulator of tumor metastasis by targeting CPEB2 in colorectal cancer. Oncotarget 8: 26858-26870, 2017.

18. Ashburner M, Ball CA, Blake JA, Botstein D, Butler H, Cherry JM, Davis AP, Dolinski K, Dwight SS, Eppig JT, et al: Gene ontology: Tool for the unification of biology. Gene Ontology Consortium. Nat Genet 25: 25-29, 2000. 
19. Ogata H, Goto S, Sato K, Fujibuchi W, Bono H and Kanehisa M: KEGG: Kyoto encyclopedia of genes and genomes. Nucleic Acids Res 27: 29-34, 1999.

20. Huang da W, Sherman BT and Lempicki RA: Systematic and integrative analysis of large gene lists using DAVID bioinformatics resources. Nat Protoc 4: 44-57, 2009.

21. Szklarczyk D, Franceschini A, Wyder S, Forslund K, Heller D, Huerta-Cepas J, Simonovic M, Roth A, Santos A, Tsafou KP, et al: STRING v10: Protein-protein interaction networks, integrated over the tree of life. Nucleic Acids Res 43: D447-D452, 2015.

22. Shannon P, Markiel A, Ozier O, Baliga NS, Wang JT, Ramage D, Amin N, Schwikowski B and Ideker T: Cytoscape: A software environment for integrated models of biomolecular interaction networks. Genome Res 13: 2498-2504, 2003.

23. John B, Enright AJ, Aravin A, Tuschl T, Sander C and Marks DS Human MicroRNA targets. PLoS Biol 2: e363, 2004.

24. Kertesz M, Iovino N, Unnerstall U, Gaul U and Segal E: The role of site accessibility in microRNA target recognition. Nat Genet 39: 1278-1284, 2007

25. Lewis BP, Burge CB and Bartel DP: Conserved seed pairing, often flanked by adenosines, indicates that thousands of human genes are microRNA targets. Cell 120: 15-20, 2005.

26. Wong $\mathrm{N}$ and Wang X: miRDB: An online resource for microRNA target prediction and functional annotations. Nucleic Acids Res 43: D146-D152, 2015.

27. Dweep H, Sticht C, Pandey P and Gretz N: miRWalk-database: Prediction of possible miRNA binding sites by 'walking' the genes of three genomes. J Biomed Inform 44: 839-847, 2011.

28. Tang Z, Li C, Kang B, Gao G, Li C and Zhang Z: GEPIA: A web server for cancer and normal gene expression profiling and interactive analyses. Nucleic Acids Res 45: W98-W102, 2017.

29. Carbon S, Ireland A, Mungall CJ, Shu S, Marshall B, Lewis S, AmiGO Hub and Web Presence Working Group: AmiGO: Online access to ontology and annotation data. Bioinformatics 25: 288-289, 2009

30. Guo Y, Bao Y, Ma M and Yang W: Identification of key candidate genes and pathways in colorectal cancer by integrated bioinformatical analysis. Int J Mol Sci 18: E722, 2017.

31. Yu JT, Tan L and Hardy J: Apolipoprotein E in Alzheimer's disease: An update. Annu Rev Neurosci 37: 79-100, 2014.

32. Borgquist S, Butt T, Almgren P, Shiffman D, Stocks T, Orho-Melander M, Manjer J and Melander O: Apolipoproteins, lipids and risk of cancer. Int J Cancer 138: 2648-2656, 2016.

33. Zamanian-Daryoush M and DiDonato JA: Apolipoprotein A-I and cancer. Front Pharmacol 6: 265, 2015.

34. Mangaraj M, Nanda R and Panda S: Apolipoprotein A-I: A molecule of diverse function. Indian J Clin Biochem 31: 253-259, 2016.

35. Peltier J, Roperch JP, Audebert S, Borg JP and Camoin L: Quantitative proteomic analysis exploring progression of colorecta cancer: Modulation of the serpin family. J Proteomics 148 139-148, 2016.

36. Sirnio P, Vayrynen JP, Klintrup K, Makela J, Makinen MJ, Karttunen TJ and Tuomisto A: Decreased serum apolipoprotein A1 levels are associated with poor survival and systemic inflammatory response in colorectal cancer. Sci Rep 7: 5374 2017.

37. Gong Y, Zhang L, Bie P and Wang H: Roles of ApoB-100 gene polymorphisms and the risks of gallstones and gallbladder cancer: A meta-analysis. PLoS One 8: e61456, 2013.

38. Zhong DN, Ning QY, Wu JZ, Zang N, Wu JL, Hu DF, Luo SY, Huang AC, Li LL and Li GJ: Comparative proteomic profiles indicating genetic factors may involve in hepatocellular carcinoma familial aggregation. Cancer Sci 103: 1833-1838, 2012.

39. Beecken WD, Ringel EM, Babica J, Oppermann E, Jonas D and Blaheta RA: Plasmin-clipped $\beta_{2}$-glycoprotein-I inhibits endothelial cell growth by down-regulating cyclin A, B and D1 and up-regulating p21 and p27. Cancer Lett 296: 160-167, 2010.

40. Jing X, Tian ZB, Gao PJ, Han NJ, Xu YH, Zhang H, Ding XL, Wang XW, Man X and Zhang CP: Lipopolysaccharide enhances beta2-glycoprotein I activation of nuclear factor $\kappa \mathrm{B}$ in liver cancer cells. Clin Lab 61: 1239-1245, 2015.

41. Huang H, Han Y, Gao J, Feng J, Zhu L, Qu L, Shen L and Shou C: High level of serum AMBP is associated with poor response to paclitaxel-capecitabine chemotherapy in advanced gastric cancer patients. Med Oncol 30: 748, 2013.

42. Rasul S, Wagner L and Kautzky-Willer A: Fetuin-A and angiopoietins in obesity and type 2 diabetes mellitus. Endocrine 42 : 496-505, 2012
43. Nangami GN, Watson K, Parker-Johnson K, Okereke KO, Sakwe A, Thompson P, Frimpong N, Ochieng J: Fetuin-A (alpha2HS-glycoprotein) is a serum chemo-attractant that also promotes invasion of tumor cells through Matrigel. Biochem Biophys Res Commun 438: 660-665, 2013.

44. Fan NJ, Kang R, Ge XY, Li M, Liu Y, Chen HM and Gao CF: Identification alpha-2-HS-glycoprotein precursor and tubulin beta chain as serology diagnosis biomarker of colorectal cancer. Diagn Pathol 9: 53, 2014.

45. Kumari S and Malla R: New insight on the role of plasminogen receptor in cancer progression. Cancer Growth Metastasis 8: 35-42, 2015.

46. Didiasova M, Wujak L, Wygrecka M and Zakrzewicz D: From plasminogen to plasmin: Role of plasminogen receptors in human cancer. Int J Mol Sci 15: 21229-21252, 2014.

47. Heit JA, Silverstein MD, Mohr DN, Petterson TM, O'Fallon WM and Melton LJ III: Risk factors for deep vein thrombosis and pulmonary embolism: A population-based case-control study. Arch Intern Med 160: 809-815, 2000.

48. Dicke C and Langer F: Pathophysiology of Trousseau's syndrome. Hamostaseologie 35: 52-59, 2015.

49. Guglietta S and Rescigno M: Hypercoagulation and complement: Connected players in tumor development and metastases. Semin Immunol 28: 578-586, 2016

50. Afshar-Kharghan V: The role of the complement system in cancer. J Clin Invest 127: 780-789, 2017.

51. Torres S, Garcia-Palmero I, Bartolome RA, FernandezAcenero MJ, Molina E, Calvino E, Segura MF and Casal JI: Combined miRNA profiling and proteomics demonstrates that different miRNAs target a common set of proteins to promote colorectal cancer metastasis. J Pathol 242: 39-51, 2017.

52. Hur K, Toiyama Y, Schetter AJ, Okugawa Y, Harris CC, Boland CR and Goel A: Identification of a metastasis-specific MicroRNA signature in human colorectal cancer. J National Cancer Ins 107: 2015.

53. Vychytilova-Faltejskova P, Pesta M, Radova L, Liska V, Daum O, Kala Z, Svoboda M, Kiss I and Slaby O: Genome-wide microRNA expression profiling in primary tumors and matched liver metastasis of patients with colorectal cancer. Cancer Genomics Proteomics 13: 311-316, 2016.

54. Abdelmaksoud-Dammak R, Chamtouri N, Triki M, Saadallah-Kallel A, Ayadi W, Charfi S, Khabir A, Ayadi L, Sallemi-Boudawara T and Mokdad-Gargouri R: Overexpression of miR-10b in colorectal cancer patients: Correlation with TWIST-1 and E-cadherin expression. Tumour Biol 39 : 1010428317695916, 2017.

55. Jiang H, Liu J, Chen Y, Ma C, Li B and Hao T: Up-regulation of mir-10b predicate advanced clinicopathological features and liver metastasis in colorectal cancer. Cancer Med 5: 2932-2941, 2016.

56. Gullu G, Karabulut $S$ and Akkiprik M: Functional roles and clinical values of insulin-like growth factor-binding protein-5 in different types of cancers. Chin J Cancer 31: 266-280, 2012.

57. Femia AP, Luceri C, Toti S, Giannini A, Dolara P and Caderni G: Gene expression profile and genomic alterations in colonic tumours induced by 1,2-dimethylhydrazine (DMH) in rats. BMC Cancer 10: 194, 2010.

58. Yu L, Lu Y, Han X, Zhao W, Li J, Mao J, Wang B, Shen J, Fan S, Wang L, et al: microRNA-140-5p inhibits colorectal cancer invasion and metastasis by targeting ADAMTS5 and IGFBP5. Stem Cell Res Ther 7: 180, 2016.

59. Schmugge M, Rand ML and Freedman J: Platelets and von Willebrand factor. Transfus Apher Sci 28: 269-277, 2003

60. O'Sullivan JM, Preston RJS, Robson T and O'Donnell JS: Emerging roles for von willebrand factor in cancer cell biology. Semin Thromb Hemost 44: 159-166, 2018.

61. Wang WS, Lin JK, Lin TC, Chiou TJ, Liu JH, Yen CC and Chen PM: Plasma von Willebrand factor level as a prognostic indicator of patients with metastatic colorectal carcinoma. World J Gastroenterol 11: 2166-2170, 2005.

62. Mochizuki S, Soejima K, Shimoda M, Abe H, Sasaki A, Okano HJ, Okano H and Okada Y: Effect of ADAM28 on carcinoma cell metastasis by cleavage of von Willebrand factor. J NatI Cancer Inst 104: 906-922, 2012.

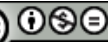

This work is licensed under a Creative Commons Attribution-NonCommercial-NoDerivatives 4.0 International (CC BY-NC-ND 4.0) License. 